\title{
Effects of Professional Toothbrushing and Instruction in the Elderly: A Randomized Trial
}

\author{
Eun-Deok Jo ${ }^{1}$, Eun-Sol $\mathrm{Kim}^{2}$, Hae-Kyung Hong ${ }^{3}$, and Gyeong-Soon $\mathrm{Han}^{1,2, \dagger}$ \\ ${ }^{1}$ Department of Dental Hygiene, College of Health Science, Gachon University, Incheon 21936, \\ ${ }^{2}$ Department of Health Science, Graduate School of Gachon University, Incheon 21936, \\ ${ }^{3}$ Department of Dental Hygiene, Kyungdong University, Wonju 26495, Korea
}

\begin{abstract}
The purpose of this study was to identify the effect of providing toothbrushing by professionals as part of oral hygiene education for the elderly. We randomly visited 12 centers in the metropolitan city and analyzed the data of 114 participants among 310 early registrants aged $>65$ years, who participated in the study at all 3 time points. The subjects were categorized into an experimental group (odd-numbered visits) and a control group (even-numbered visits). Oral hygiene practices were provided for both experimental and control groups, but professional toothbrushing was performed in a different manner in the experimental group. Differences in plaque index (PI) according to the subjects' general characteristics and oral health status were assessed using the t-test, and the effect of PI difference between the experimental and control groups was assessed by repeated measure two-way analysis of variance. A stepwise multiple regression analysis was used to analyze factors affecting the PI. At baseline, the mean overall PI was 61.82. In both experimental and control groups, the PI significantly reduced from the baseline $(p<0.01)$. At 5 weeks, the experimental group showed a decrease of 27.16 points from the baseline, and the 10-week PI was similar to the 5-week PI. The control group showed a decrease of 14.87 points from the baseline, and the PI increased by 5.74 points at 10 weeks. Pl-related factors were gender and self-xerostomia. The PI was lower in the female group and the group with self- xerostomia $(p<0.01)$. It is important to select an appropriate method to educate elderly subjects on proper removal of dental plaque and to habituate correct behavior, which requires inclusion of a direct toothbrushing intervention by a specialist.
\end{abstract}

Key Words: Dental plaque, Elderly, Professional toothbrushing

\section{Introduction}

Elderly people experience a gradual decline in physical function with age and are more likely to develop chronic diseases. In the oral environment, the movement of the tongue and lips reduces, the amount of saliva secreted decreases, discomfort increases, and the likelihood of oral disease development increases. Since oral health in the elderly is closely related to their general health ${ }^{1)}$, oral hygiene management is essential to maintain overall health. Bacterial colonization in the oral environment begins with deterioration of oral hygiene ${ }^{2}$. Microorganisms colonized on the tooth surface form dental plaques and cause dental caries and periodontal disease ${ }^{3)}$, leading to tooth loss and halitosis. In elderly individuals, the poor masticatory capacity resulting from oral disease causes malnutrition and a poor quality of life ${ }^{4}$. Therefore, it is necessary to prevent the growth of potential pathogens in the oral environment by removing dental plaque and food debris attached to the tooth surface. Dental plaque control refers to the methods to prevent the formation of dental plaque on tooth surfaces or to remove the attached dental plaque. The most-basic method for removal of dental plaque is brushing with a toothbrush, dental floss, and an interdental toothbrush. However, many people tend to brush their teeth habitually rather than in the correct manner. Since the habitual form of toothbrushing cannot clean the teeth appropriately, it is necessary to correct the 
toothbrushing method. In addition, when self-management and professional management are performed in parallel, it is possible to effectively manage dental plaque. Professional management involves identifying the subject's brushing habits and oral health status and thereafter providing plaque-control instructions and professional toothbrushing $(\mathrm{PT})^{5)}$. PT uses the "Watanabe method" with a two-row brush; since toothbrushing is performed directly on the subject, this method allows for excellent plaque removal ${ }^{6)}$. In comparison with the "modified Bass method," the Watanabe method shows better plaque removal, thus improving oral hygiene ${ }^{7)}$. In addition, gingival massage in the Watanabe method has been reported to improve keratinization and increase resistance to bacteria, thus improving immunity associated with the periodontal tissue and preventing oral disease ${ }^{8)}$. Therefore, an important step in providing plaque-control instructions is to help patients understand how the toothbrush should make contact with the tooth surface by performing PT. For successful oral health education, technical methods should be demonstrated not only using educational dental model but also directly on the oral cavity of individual subjects ${ }^{9)}$. Eom et al. ${ }^{10}$ ) reported that training for 3 to 4 sessions is required to reach a certain level of dental plaque-management ability. In particular, elderly people need more delicate and repetitive demonstrations than normal adults due to their slow wrist movements when brushing. Hence, the purpose of this study was to identify the effect of providing toothbrushing by professionals as a part of oral hygiene education for the elderly and to use this knowledge in the development of oral hygiene-education programs.

\section{Materials and Methods}

\section{Subjects}

This study was conducted from April 4, 2016 to June 11, 2016, after obtaining approval from the Institutional Review Board of Gachon University (IRB No. 1044396201511-HR-057-02). To determine the sample size, $\mathrm{G}^{*}$ Power 3.1. software (Heinrich-Heine-University Dusseldorf, Dusseldorf, Germany) was used. To calculate the sample size, alpha error probability and power were set at 0.05 and 0.8 . In addition, the effect size was set at 0.25 on the basis of Cohen's methods ${ }^{11)}$. With these parameters, a total sample size of 86 patients was required. The subjects were sufficiently explained about the purpose and process of this study. Those who agreed to participate in this study completed the consent form and were included as research participants. The researcher approached 12 senior centers in the metropolitan city to recruit participants, and the study was started with 204 subjects in the experimental group and 106 subjects in the control group. The subjects were categorized into an experimental group (oddnumbered visits) and a control group (even-numbered visits). However, data of only 114 participants (experimental group, 84; control group, 30) who participated in the study at all 3 time points were finally analyzed (Fig. 1).

\section{Methods}

\section{1) Questionnaire interview}

Using a questionnaire, the researchers asked subjects' information about their age, gender, education level, gingival bleeding, self-assessed xerostomia, and selfassessed halitosis, the researchers recorded the responses of the subjects. The response options for oral health status parameters were "Yes" or "No."

\section{2) Oral assessment}

The number of remaining teeth was evaluated by direct oral examination and assessed by one dentist. The number

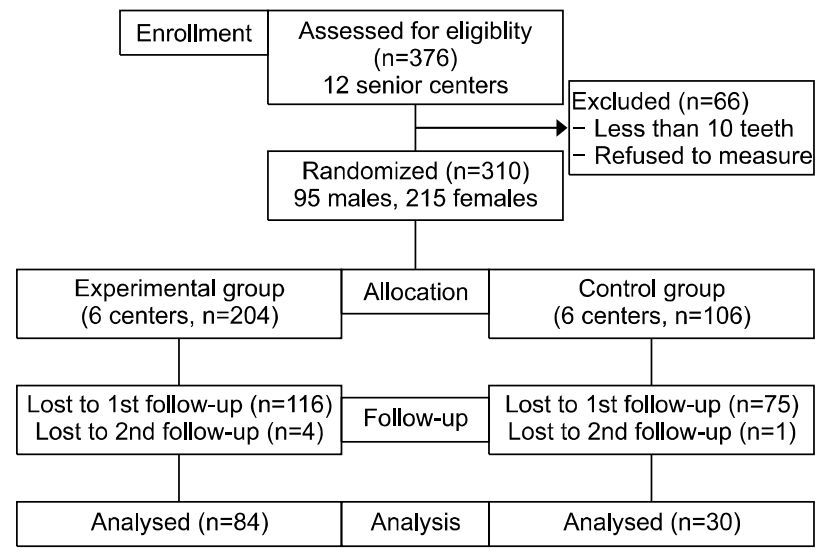

Fig. 1. Study enrollment flow. 
of remaining teeth was calculated by counting natural teeth and prosthetic teeth and excluding pontic teeth that could still perform masticatory functions in the oral cavity. However, the third molar and teeth with a degree of mobility $\geq 2$ were excluded.

The plaque index (PI) was assessed by one dental hygienist and measured using the O'Leary index ${ }^{12)}$. The O'Leary index is useful for quantitatively measuring oral hygiene in individuals and is a reliable and reproducible method. All teeth in the oral cavity are divided into 4 categories - mesial, distal, buccal, and lingual - to evaluate the presence of dental plaque on tooth surfaces. After applying the disclosing agent (2 Tone Disclosing Solution $^{\text {TM}}$; Young Dental Manufacturing, Earth City, MO, USA) to visualize tooth-surface plaque, the researcher evaluated the PI. To assign points to the system, non-adherent plaques were classified as " 0 " and adherent plaques as " 1 " (at this time, missing teeth were excluded, and artificial teeth were included). The percentage was calculated by dividing the sum of the number of plaqueattached tooth surfaces by the total number of tooth surfaces.

\section{3) Oral hygiene education}

To motivate the subjects about oral hygiene management, the researchers took a plaque of the subject's oral cavity and confirmed it with a phase-contrast microscope. The image on the screen showed the relation between the oral microorganisms' morphology/movement and oral disease and emphasized the need for plaque control.

Plaque-control instructions were provided in both experimental and control groups. Education on toothbrushing involved indirectly instructing the participants according to the Watanabe brushing method by using an educational dental model and toothbrush. In addition, all subjects were educated on the usage of an interdental toothbrush and dental floss. After the instructions, equivalent toothbrushes, toothpaste, interdental toothbrushes, and dental floss were distributed to all subjects.

PT performed differently to only the experimental group. Subjects who used a disclosing agent in the oral assessment checked the dental plaques with a mirror. Dental hygienists performed brushing directly using the Watanabe method in the experimental group. The subjects were placed in the supine position and asked to watch the entire process through a hand-held mirror under fluorescent lamps, and the educator performed brushing at the back position (12 o'clock location) of the subject. The toothbrush had 4 rows of fine bristles instead of 2 rows to allow the subjects to use the method with a toothbrush they could easily purchase. After brushing, the subjects directly washed their mouth with water and checked the teeth cleanliness with a mirror. This procedure was performed three times at intervals of 5 weeks, and phase-contrast microscopy was performed at the first visit. The research process is outline in Fig. 2.

\section{4) Statistical analysis}

Data were analyzed using PASW Windows ver. 18.0 (IBM Co., Armonk, NY, USA). Differences in PI according to the subjects' general characteristics and oral health status were assessed using the t-test, and the effect of a PI decrease in the experimental and control groups was assessed by repeated measure two-way analysis of

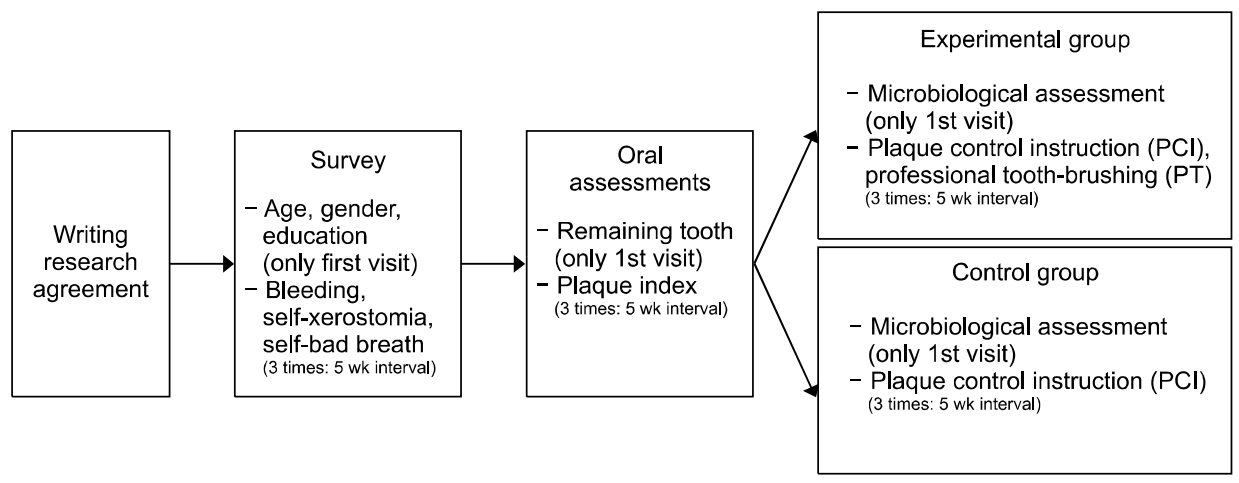

Fig. 2. The research process. 
variance. A stepwise multiple regression analysis was used to analyze factors affecting the PI. For regression analysis, the PI was based on the experimental group's PI at 10 weeks. Statistical significance was set at $\mathrm{p}<0.05$.

\section{Results}

\section{Differences in Pl according to general characteristics and oral health status}

At the beginning of the study, the total mean PI of the subjects was 61.82 (Table 1). The PI differed according to the presence of gingival bleeding $(p<0.05)$. The mean PI of the group with gingival bleeding was 67.37 and that of the group without gingival bleeding was 58.67.

\section{Effect of PI decrease in the experimental and control groups}

Table 2 shows the effect of PI decrease in the experimental and control groups. There was no statistically significant interaction between the time points and groups $(\mathrm{p}=0.062)$, but the difference in time was significant $(p=0.001)$ and the difference between the experimental group and the control group was significant $(p=0.047)$. Both groups showed a significant decrease in PI compared to the baseline. The experimental group showed a decrease of 27.16 points from the baseline at 5 weeks, and the PI at 10 weeks was similar to that at 5 weeks. In the control group, the PI at 5 weeks decreased by 14.87 points from the baseline, and the PI at 10 weeks increased by 5.74 points from that at 5 weeks.

\section{Stepwise multiple regression for PI-related factors}

Table 3 shows the results of stepwise multiple regression analysis with all the characteristics related to the subject as independent variables and the PI at 10 weeks in the experimental group as a dependent variable. Our analysis showed that the PI-related factors were gender and self-xerostomia. The PI was lower in the female group and the group with self-xerostomia $(p<0.01)$. The explanatory power of the regression model was $51.7 \%$.
Table 1. Differences in Plaque Index according to General Characteristics and Oral Health Status

\begin{tabular}{lccc}
\hline \multicolumn{1}{c}{ Variable } & $\begin{array}{c}\text { No. of } \\
\text { participants }\end{array}$ & Baseline & p-value \\
\hline General characteristics & & & \\
Age (y) & & & 0.820 \\
$\quad<73$ & 27 & $61.12 \pm 21.71$ & \\
$\quad \geq 73$ & 87 & $62.12 \pm 22.62$ & \\
Gender & & & 0.958 \\
$\quad$ Male & 33 & $61.99 \pm 23.14$ & \\
Female & 81 & $61.75 \pm 22.05$ & \\
Education & & & 0.874 \\
$\quad$ Illiteracy & 33 & $61.62 \pm 20.34$ & \\
$\quad \geq$ Elementary school & 45 & $62.41 \pm 22.65$ & \\
Oral health status & & & \\
Remaining tooth (ea) ${ }^{\mathrm{a}}$ & & & 0.700 \\
$\quad<18$ & 42 & $63.36 \pm 25.72$ & \\
$\quad \geq 18$ & 72 & $61.32 \pm 21.16$ & \\
Gingival bleeding & & & 0.040 \\
$\quad$ Absence & 81 & $58.67 \pm 21.38$ & \\
Present & 33 & $67.37 \pm 22.95$ & \\
Self-xerostomia & & & 0.121 \\
Absence & 42 & $57.68 \pm 21.58$ & \\
Present & 72 & $64.24 \pm 22.44$ & \\
Self-bad breath & & & 0.462 \\
Absence & 69 & $60.58 \pm 22.71$ & \\
$\quad$ Present & 45 & $63.65 \pm 21.67$ & \\
Total & 114 & $61.82 \pm 22.56$ & \\
\hline Valus are present & & & \\
\hline
\end{tabular}

Values are presented as number only or mean \pm standard deviation.

p-values obtained from the t-test.

${ }^{\mathrm{a}}$ It's divided into mid-level number.

Table 2. Effect of Plaque Index Decrease in the Experimental and Control Groups

\begin{tabular}{cccc}
\hline Group & Baseline & $\begin{array}{c}5 \mathrm{wk} \\
\text { after }\end{array}$ & $\begin{array}{c}10 \mathrm{wk} \\
\text { after }\end{array}$ \\
\hline $\begin{array}{c}\text { Experimental } \\
\text { group }(\mathrm{n}=84)\end{array}$ & $61.81 \pm 22.33$ & $34.65 \pm 18.83$ & $34.39 \pm 24.93$ \\
Control group $(\mathrm{n}=30)$ & $61.83 \pm 22.41$ & $46.96 \pm 23.33$ & $52.70 \pm 20.59$ \\
p-value Time & & $0.001^{\mathrm{a}}$ & \\
Group & & $0.047^{\mathrm{a}}$ & \\
Time $\times$ Group & & $0.062^{\mathrm{a}}$ & \\
\hline
\end{tabular}

Values are presented as mean \pm standard deviation.

${ }^{a}$ Repeated measure two-way ANOVA $(\mathrm{p}<0.05)$.

\section{Discussion}

At baseline of this study, the mean dental plaque score in the entire study population was 61.82 , with scores of 61.81 in the experimental group and 61.83 in the control 
Table 3. Stepwise Multiple Regression for Plaque Index (PI)-Related Factors

\begin{tabular}{lccccc}
\hline \multicolumn{1}{c}{ Independent variable } & $\mathrm{B}$ & $\mathrm{SD}$ & $\beta$ & $\mathrm{t}$ & $\mathrm{p}$-value \\
\hline Gender (male=0, female=1) & -20.563 & 6.079 & -0.579 & -3.383 & 0.004 \\
Self-xerostomia (absence=0, present $=1)$ & -22.452 & 6.398 & -0.601 & -3.510 & 0.003 \\
$\mathrm{~F}=10.109(\mathrm{p}<0.01), \mathrm{R}^{2}=0.574$, adjusted $\mathrm{R}^{2}=0.517$ & & & &
\end{tabular}

Dependent variable: PI of 10 weeks on experimental group.

Excluded variables: age $(<73$ years $=0, \geq 73$ years $=1)$, education (illiteracy $=0$, elementary school or more $=1)$, remaining teeth $(<18$ ea $=0,18$ ea $=1)$, gingival bleeding $($ absence $=0$, present $=1)$, self-bad breath $($ absence $=0$, present $=1)$.

SD: standard deviation.

group. The PI differed with regard to the presence of gingival bleeding. The dental plaque has the characteristics of a biofilm and causes oral diseases such as dental caries and periodontal disease ${ }^{13)}$. In Kim's study ${ }^{14)}$, plaque management by toothbrushing in periodontitis patients significantly decreased the gingival bleeding index after 6 weeks from the baseline (from 3.8 to 1.9). Therefore, physical removal of the dental plaque through proper toothbrushing before the formation of calculus is an important factor in preventing early gingivitis, which causes gingival bleeding. Various studies have aimed to educate and motivate correct toothbrushing habits. Bakdash $^{15)}$ found that motivation induces interest and concern, which leads to attitude and behavioral changes. Fisher et al. ${ }^{16)}$ suggested that the curriculum for behavioral change should include teaching specific behaviors and techniques and demonstration of the techniques. In addition, Hoogstraten and Moltzer ${ }^{17)}$ compared brushing training methods and found that direct brushing training was more effective than indirect brushing training. Therefore, in the process of PT in present study, the researcher presented the subjects' oral status to them through the mirror and analyzed the effects of the PT intervention by repeating it 3 times. As a result, PI of the experimental group that received PT decreased by 27.16 points at the second visit and remained steady until the third visit; however, in the control group, the PI decreased by 14.87 points at the second visit and increased by 5.74 points at the third visit. Thus, oral hygiene education involving PT was an important factor in reducing PI and maintaining the reduced PI. The effects of professional intervention and repetitive brushing training are reported in this study and other previous studies. In an assessment of brushing education among elderly subjects ${ }^{18)}$, the periodontal bleeding index and oral hygiene-management ability index showed a significant improvement after repeated PT and education on interdental brushing, regardless of the presence of chronic diseases. In an adult population, An et al. ${ }^{19)}$ showed that the patient hygiene performance index improved better in individual toothbrushing education groups after using a disclosing solution than education group used dental model. In a study that repeatedly educated college students on PT, the patient hygiene performance index decreased significantly ${ }^{20)}$. Thus, to maintain oral health, direct intervention of professionals in oral hygiene education is considered effective for improving the will and ability to remove dental plaque in subjects.

Factors affecting the PI in the experimental group were analyzed, and the related factors were gender and selfxerostomia. PI was lower in women than in men. Therefore, men should be taught more effective ways or provided with more time to understand and follow the contents of the training program. In addition, among married couples, the female partner may be able to help her spouse better understand the method. PI was lower in the group self-xerostomia than in the group without self-xerostomia. It is considered that elderly people who are good at brushing are experiencing more of selfxerostomia. Our results showed that when practicing toothbrushing for elderly people, the specialists need to conduct additional training such as oral functionimprovement exercises or diet modification to alleviate oral dryness.

This study, conducted by visiting senior centers in the metropolitan area, showed significant differences in the subjects' participation because the process was conducted 3 times at 5-week intervals. Thus, bias of participants in 
the experimental and control groups limits generalization of the results, and further study is required to obtain stratified probability samples.

The present results suggest that it is important to select an appropriate method to educate subjects about removing dental plaque properly and to habituate correct behavior. It is necessary to include a direct toothbrushing intervention by a specialist for this purpose. Therefore, a program including PT must be developed for oral hygiene education.

In conclusion, oral hygiene education thus far focuses on education for the majority population or indirect education. However, this study confirmed that the oral hygiene-education programs for the elderly should include direct toothbrushing by professionals and periodic repetitive education

\section{Acknowledgements}

The authors would like to thank all the participants for their participation in the study. This work was supported by the Gachon University research fund of 2016 (GCU-2016-0202).

\section{References}

1. Budtz-Jørgensen E, Chung JP, Rapin CH: Nutrition and oral health. Best Pract Res Clin Gastroenterol 15: 885-896, 2001. https://doi.org/10.1053/bega.2001.0247

2. Palmer LB, Albulak K, Fields S, Filkin AM, Simon S, Smaldone G: Oral clearance and pathogenic oropharyngeal colonization in the elderly. Am J Respir Crit Care Med 164: 464-468, 2001. https://doi.org/10.1164/ajrccm.164.3.2008149

3. Lang NP, Mombelli A, Attström R: Oral biofilms and calculus. In: Lindhe J, Lang NP, Karring T, eds. Clinical periodontology and implant dentistry. 5th ed. Blackwell Munksgaard, Oxford, pp.183-206, 2008.

4. Marik PE, Kaplan D: Aspiration pneumonia and dysphagia in the elderly. Chest 124: 328-336, 2003. https://doi.org/10.1378/chest.124.1.328

5. Choi HN, Lim SR, Cho YS: The dental biofilm reduction effect \& control difficulty level of university students through dental biofilm control program. J Dent Hyg Sci 12: 39-44,
2012.

6. Chang KW: Professional dental plaque management: watanabe method. J Korean Dent Assoc 45: 21-24, 2007.

7. Poyato-Ferrera M, Segura-Egea JJ, Bullón-Fernández P: Comparison of modified bass technique with normal toothbrushing practices for efficacy in supragingival plaque removal. Int J Dent Hyg 1: 110-114, 2003. https://doi.org/10.1034/j.1601-5037.2003.00018.x

8. Lee BJ: Professional oral health care program using toothpick method toothbrushing. J Korean Dent Assoc 47: 272-281, 2009.

9. Choi MS, Kim DK: Reviews of literature on dental plaque control and oral hygiene education in Korea. J Dent Hyg Sci 17: 87-98, 2017. https://doi.org/10.17135/jdhs.2017.17.2.87

10. Eom MR, Jeong DB, Park DY: Enhancement of plaque control score following individualized repeated instruction. $\mathrm{J}$ Korean Acad Dent Health 33: 10-18, 2009.

11. Cohen J: Statistical power analysis for the behavioral sciences. 2nd ed. Lawrence Erlbaum, Hillsdale, pp.24-26, 1988.

12. O'Leary TJ, Drake RB, Naylor JE: The plaque control record. J Periodontol 43: 38, 1972. https://doi.org/10.1902/jop.1972.43.1.38

13. Briner WW: Plaque in relation to dental caries and periodontal disease. Int Dent J 21: 293-301, 1971.

14. Kim SH: The effect of plaque control (tooth brushing instruction) for oral health improvement on periodontitis patients. J Korean Soc Dent Hyg 11: 293-301, 2011.

15. Bakdash MB: Patient motivation and education: a conceptual model. Clin Prev Dent 1: 10-14, 1979.

16. Fisher EB, Brownson CA, O’Toole ML, Shetty G, Anwuri VV, Glasgow RE: Ecological approaches to self-management: the case of diabetes. Am J Public Health 95: 1523-1535, 2005. https://doi.org/10.2105/AJPH.2005.066084

17. Hoogstraten J, Moltzer G: Effects of dental health care instruction on knowledge, attitude, behavior and fear. Community Dent Oral Epidemiol 11: 278-282, 1983. https://doi.org/10.1111/j.1600-0528.1983.tb01893.x

18. Cho MM, Lee YH, Kim JB, Lee JH: Promotion of periodontal health through professional toothbrushing and education on the use of the interdental brush in the elderly. $\mathbf{J}$ Korean Acad Dent Health 37: 123-140, 2013. https://doi.org/10.11149/jkaoh.2013.37.3.132 
19. An HH, Lee MH, Seo HJ: Oral hygiene performance ability according to toothbrushing instruction. J Korea Soc Health Inform Stat 37: 43-52, 2012.

20. Kim YH, Kim YG, Yoo JA, Park JE: The effect of the cariview test and professional brushing training on the reduction of PHP index of dental hygiene students. J Korean Soc Dent Hyg 17: 993-1001, 2017.

https://doi.org/10.13065/jksdh.2017.17.06.993 